\title{
Pacific
}

Journal of

Mathematics

\section{SIGMA THEORY AND TWISTED CONJUGACY CLASSES}

DaCiberg Gonçalves and Dessislava Hristova Kochloukova 


\title{
SIGMA THEORY AND TWISTED CONJUGACY CLASSES
}

\author{
Daciberg GonçAlves AND Dessislava Hristova Kochloukova
}

\begin{abstract}
Using Sigma theory we show that for large classes of groups $G$ there is a subgroup $H$ of finite index in $\operatorname{Aut}(G)$ such that for $\varphi \in H$ the Reidemeister number $R(\varphi)$ is infinite. This includes all finitely generated nonpolycyclic groups $G$ that fall into one of the following classes: nilpotent-by-abelian groups of type $F P_{\infty}$; groups $G / G^{\prime \prime}$ of finite Prüfer rank; groups $G$ of type $F P_{2}$ without free nonabelian subgroups and with nonpolycyclic maximal metabelian quotient; some direct products of groups; or the pure symmetric automorphism group. Using a different argument we show that the result also holds for 1-ended nonabelian nonsurface limit groups. In some cases, such as with the generalized Thompson's groups $F_{n, 0}$ and their finite direct products, $H=\operatorname{Aut}(G)$.
\end{abstract}

\section{Introduction}

We study the Reidemeister number $R(\varphi)$ for elements $\varphi$ of subgroups of finite index in $\operatorname{Aut}(G)$ for large classes of groups $G$. The Reidemeister number $R(\varphi)$ counts the twisted conjugacy classes $\left\{g a \varphi(g)^{-1}\right\}_{g \in G}$ for $a \in G$. The study of $R(\varphi)$ began in the Nielsen-Reidemeister fixed point theory. Following [Taback and Wong 2007], we say a group $G$ has the property $R_{\infty}$ if $R(\varphi)$ is infinite for every automorphism $\varphi$ of $G$. Many classes of groups have the property $R_{\infty}$ : nonelementary Gromov hyperbolic groups [Levitt and Lustig 2000; Fel'shtyn 2001], nonabelian generalized Baumslag-Solitar groups [Levitt 2007], saturated weakly branch groups (including the Grigorchuk group and the Gupta-Sidki group) [Fel'shtyn et al. 2008a], and the Thompson's group $F$ [Bleak et al. 2008].

Fel'shtyn et al. [2008b] showed that for a finitely generated polyfree group $G$ with nonzero Euler characteristic, there is a subgroup $H$ of finite index in $\operatorname{Aut}(G)$ such that the Reidemeister number is infinite for every element of $H$.

In Sections 4a and 4c, we will show that to the groups in the list above that have the $R_{\infty}$ property, we can add the generalized Thompson's groups $F_{n, 0}$ and any finite direct product of such groups (with possibly different $n$ ).

MSC2010: primary 20F65, 20J05, 55M20; secondary 20E45.

Keywords: Reidemeister class, Thompson group, Sigma theory, automorphism of groups, $R_{\infty}$

property, limit group.

Both authors are partially supported by "bolsa de produtividade de pesquisa" from CNPq, Brazil. 
Furthermore we study large classes of groups $G$ where it is possible to show that there is a subgroup $H$ of finite index in $\operatorname{Aut}(G)$ such that $R(\varphi)$ is infinite for every $\varphi \in H$. These are all finitely generated nonpolycyclic groups $G$ such that

(1) $G$ is nilpotent-by-abelian of type $F P_{\infty}$;

(2) $G / G^{\prime \prime}$ is of finite Prüfer rank but not polycyclic;

(3) $G$ is of type $F P_{2}$ with no free nonabelian subgroups and its maximal metabelian quotient is not polycyclic;

(4) $G$ is some direct product of groups;

(5) $G$ is the pure symmetric automorphism group;

(6) $G$ is a Houghton group $H_{n}$.

The proofs of these results, found in Section 4, rely on the structure of the invariant $\Sigma^{1}(G)$ for the groups $G$ described above. In the preliminaries we detail the properties of this invariant. It appeared first in the study of finitely presented metabelian groups $G$ [Bieri and Strebel 1980] and was later defined for any finitely generated group $G$ [Bieri et al. 1987]. The geometric invariant $\Sigma^{1}(G)$ is invariant under the action of the automorphism group of $G$, which is fundamental for the proofs. In many cases we do not know the full automorphism group $\operatorname{Aut}(G)$, but using Sigma theory we can nevertheless obtain sufficient information on how $\operatorname{Aut}(G)$ can act on the abelianization of $G$.

In the final section we show that to the list above we can add

(7) $G$ is any 1-ended nonabelian nonsurface limit group.

Orientable surface groups of genus at least 2 and nonorientable surface groups of genus at least 3 are Gromov hyperbolic [Gersten 1999, 3.17.5], and hence as mentioned before have $R_{\infty}$. Nonorientable surface groups of genus 1 or 2 are not limit groups since in the first case they have torsion and in the second they are virtually abelian but not abelian.

Limits groups appeared in the solution of the Tarski problem by O. Kharlampovich, A. Myasnikov, and independently by Z. Sela. The definition of limit groups was suggested by Z. Sela, and it turned out that the class of limit groups coincides with the class of finitely generated, fully residually free groups studied by G. Baumslag, O. Kharlampovich, A. Myasnikov, and V. Remeslenikov. The class of limit groups includes finitely generated free groups, finite rank free abelian groups, and surface groups of Euler characteristic at most -2 . Limit groups are CAT(0) [Alibegović and Bestvina 2006]. Unfortunately we cannot use $\Sigma^{1}(G)$ to study the Reidemeister numbers for a limit group $G$ because the set $\Sigma^{1}(G)$ is empty [Kochloukova 2010]. Instead we use the structure theorem of $\operatorname{Aut}(G)$ for 1-ended limit groups $G$ given in [Bumagin et al. 2007]. 


\section{Preliminaries}

2a. Sigma invariants for finitely generated groups. Let $R$ be an associative ring with 1. All modules and actions in this paper are left ones. For an $R$-module $M$, we say that $M$ has type $F P_{m}$ if there is a projective resolution

$$
\cdots \rightarrow P_{i} \rightarrow P_{i-1} \rightarrow \cdots \rightarrow P_{0} \rightarrow M \rightarrow 0
$$

such that all projective modules $P_{i}$ are finitely generated for $i \leq m$. A group $G$ is of type $F P_{m}$ if the trivial module $\mathbb{Z}$ is of type $F P_{m}$ as a $\mathbb{Z} G$-module. Every group is of type $F P_{0}$, and $G$ is of type $F P_{1}$ if and only if $G$ is finitely generated. If $G$ is finitely presented (that is, there is a $K(G, 1)$ with a finite 2-skeleton), then $G$ is of type $\mathrm{FP}_{2}$. The converse does not hold [Bestvina and Brady 1997].

The homological invariants $\Sigma^{m}(G, \mathbb{Z})$ were defined in their current form in [Bieri and Renz 1988], but in the case that $m=1$ and $G$ is metabelian, they can be traced back (under different notation) to [Bieri and Strebel 1980], where they played a crucial part in classifying finitely presented metabelian groups.

For a general finitely generated group $G$, define the character sphere

$$
S(G)=\left(\operatorname{Hom}_{\mathbb{Z}}(G, \mathbb{R}) \backslash\{0\}\right) / \sim,
$$

where $\mathbb{R}$ is considered a group via addition, and $\chi_{1} \sim \chi_{2}$ if there is a positive real number $r$ such that $r \chi_{1}=\chi_{2}$. Write $[\chi]$ for the class of $\chi$ in $S(G)$, and define

$$
\Sigma^{m}(G, \mathbb{Z})=\left\{[\chi] \in S(G) \mid \mathbb{Z} \text { is of type } F P_{m} \text { as a } \mathbb{Z} G_{\chi} \text {-module }\right\},
$$

where $G_{\chi}$ is the monoid $\{g \in G \mid \chi(g) \geq 0\}$. By definition,

$$
\Sigma^{m}(G, \mathbb{Z})^{c}=S(G) \backslash \Sigma^{m}(G, \mathbb{Z}) .
$$

It is known that $\Sigma^{m}(G, \mathbb{Z})$ is always an open subset of $S(G)$ and can be empty (as in the case of a noncyclic free group). If $\Sigma^{m}(G, \mathbb{Z})$ is not empty, then $G$ is of type $F P_{m}$, but the converse does not hold (for example, when $G$ is noncyclic free). The following theorem shows that the invariants $\Sigma^{m}(G, \mathbb{Z})$ are responsible for the homological type of subgroups of $G$ that contain the commutator.

Theorem 2.1 [Bieri and Renz 1988, Theorem B]. If $N$ is a subgroup of $G$ that contains the commutator and $G$ is of type $F P_{m}$, then $N$ is $F P_{m}$ if and only if $S(G, N):=\{[\chi] \in S(G) \mid \chi(N)=0\} \subseteq \Sigma^{m}(G, \mathbb{Z})$.

There is a homotopical version $\Sigma^{m}(G)$ of $\Sigma^{m}(G, \mathbb{Z})$. In the dimension $m=$ 1 the invariants coincide, that is, $\Sigma^{1}(G, \mathbb{Z})=\Sigma^{1}(G)$, and in general $\Sigma^{m}(G) \subseteq$ $\Sigma^{m}(G, \mathbb{Z})$, but the inclusion can be strict for $m \geq 2$ [Meier et al. 1998]. As before, the superscript $c$ means complement in $S(G)$. 
In general it is hard to calculate all the invariants (both homological and homotopical versions for arbitrary dimension $m$ ). In this paper we apply some structural results about $\Sigma^{1}(G)^{c}$ to the theory of twisted conjugacy classes.

2b. A Sigma invariant for a metabelian group. In this section we discuss the original Bieri-Strebel invariant [1980]. This is the form that will be used in the proof of Theorem 4.6.

Let $Q$ be a finitely generated abelian group, and let $A$ be a finitely generated left $\mathbb{Z} Q$-module. If not otherwise stated, all modules in this paper are left ones. Then

$$
\Sigma_{A}(Q)=\left\{[\chi] \in S(Q) \mid A \text { is finitely generated as a } \mathbb{Z} Q_{\chi} \text {-module }\right\}
$$

and

$$
\Sigma_{A}^{c}(Q)=S(Q) \backslash \Sigma_{A}(Q) .
$$

Bieri and Strebel $[1981,(1.5)]$ showed that $\Sigma_{A}^{c}(Q)$ is additive, that is, if

$$
0 \rightarrow A_{1} \rightarrow A \rightarrow A_{2} \rightarrow 0
$$

is a short exact sequence of finitely generated $\mathbb{Z} Q$-modules, then

$$
\Sigma_{A}^{c}(Q)=\Sigma_{A_{1}}^{c}(Q) \cup \Sigma_{A_{2}}^{c}(Q) .
$$

It follows from the definitions that if $\chi$ is a real character of a finitely generated metabelian group $G$ such that $A$ is an abelian normal subgroup of $G$ with $Q=G / A$ abelian, and if $\chi(A) \neq 0$, then $[\chi] \in \Sigma^{1}(G)$. If $\chi(A)=0$, then $[\chi] \in \Sigma^{1}(G)^{c}$ if and only if $[\chi] \in \Sigma_{A}^{c}(Q)$ (here we consider $\chi$ as a character of $Q$ since $\chi(A)=0$ ).

Let $I$ be the annihilator of $A$ in $\mathbb{Z} Q$, that is, $I=\{\lambda \in \mathbb{Z} Q \mid \lambda A=0\}$, and let $P_{1}, \ldots, P_{s}$ be the minimal associated primes of $A$ as a $\mathbb{Z} Q$-module. Then we have by [Bieri and Strebel 1981, (1.12)]

$$
\Sigma_{A}^{c}(Q)=\Sigma_{\mathbb{Z} Q / I}^{c}(Q)=\bigcup_{1 \leq i \leq s} \Sigma_{\mathbb{Z} Q / P_{i}}^{c}(Q) .
$$

2c. Twisted conjugacy classes. Let $\varphi: G \rightarrow G$ be a group endomorphism. The set of equivalence classes $\left\{g a \varphi(g)^{-1}\right\}_{g \in G}$ for $a \in G$ is denoted by $\mathscr{R}[\varphi]$. The cardinality of $\mathscr{R}[\varphi]$ is denoted by $R(\varphi)$ and called the Reidemeister number of $\varphi$.

Let $H$ be a normal subgroup of a group $G$ invariant under an endomorphism $\varphi$. Denote by $\varphi^{\prime}$ the restriction of $\varphi$ on $H$ and by $\bar{\varphi}$ the endomorphism induced by $\varphi$ on $Q=G / H$. Then by [Gonçalves 1998] and [Gonçalves and Wong 2003, (2.2)], there is an exact sequence of sets

$$
\mathscr{R}\left[\varphi^{\prime}\right] \rightarrow \mathscr{R}[\varphi] \rightarrow \mathscr{R}[\bar{\varphi}] \rightarrow 0
$$


By [Jiang 1983, page 33], if $G / H$ is abelian, nontrivial, torsion-free, and the map $\operatorname{id}_{Q}-\bar{\varphi}$ is not surjective (that is, $\bar{\varphi}$ is a linear operator with eigenvalue 1 ), then $R(\bar{\varphi})$ is infinite and consequently $R(\varphi)$ is infinite.

\section{General results}

A point $[\chi]$ of the character sphere $S(G)$ is called a discrete point (or a rational point) if $[\chi]=[\mu]$ for some character $\mu$ such that $\operatorname{Im}(\mu)=\mathbb{Z}$. In this case we call $\chi$ discrete or rational.

Lemma 3.1. Let $G$ be a finitely generated group such that

$$
\Sigma^{1}(G)^{c}=\left\{\left[\chi_{1}\right], \ldots,\left[\chi_{m}\right]\right\}
$$

is finite, nonempty, and contains only discrete points, and let $\varphi$ be an automorphism of $G$. Let $N=\bigcap_{i} \operatorname{Ker}\left(\chi_{i}\right)$, let $V=\operatorname{Hom}_{\mathbb{Z}}(G / N, \mathbb{R})$, and let $\theta$ be the element of $\operatorname{End}_{\mathbb{Z}}(V)$ induced by $\varphi$.

Then $\theta$ permutes the images $\bar{\chi}_{i}$ of $\chi_{i}$ in $V$, where every $\chi_{i}$ is chosen so that the coordinates of $\bar{\chi}_{i}$ are integers with maximal common divisor 1.

Proof. Since $\Sigma^{1}(G)^{c}$ is invariant under automorphisms, we have $\theta\left(\bar{\chi}_{i}\right)=r_{i} \bar{\chi}_{\pi(i)}$, where $\pi$ is some element of the symmetric group $S_{m}$ and the $r_{i}$ are positive real numbers. By assumption, the coordinates of the entries of $\bar{\chi}_{i}$ (with respect to some fixed basis of the free abelian group $G / N$ ) are coprime integers. Then every $r_{i}$ is a positive integer.

We aim to show that $r_{1}=\cdots=r_{m}=1$. Indeed for $k=m$ !, we have $\theta^{k}\left(\bar{\chi}_{i}\right)=\lambda_{i} \bar{\chi}_{i}$ for some positive integer $\lambda_{i}$ divisible by $r_{i}$ for every $i$. Now $\varphi$ is invertible and $N$ is a characteristic subgroup of $G$, so $\theta$ is an automorphism of $V$, and so $\theta^{k}$ is invertible. Furthermore $X=\left\{\bar{\chi}_{i}\right\}_{1 \leq i \leq m}$ spans $V$. Then by picking up a subset $Y$ of $X$ that is a basis of $V$, we see that the operator $\theta^{k}$ is diagonalizable with eigenvalues $\lambda_{i}$ (note that a fixed $\bar{\chi}_{i}$ can always be included in some $Y$ ). Then $\prod_{\bar{\chi}_{i} \in Y} \lambda_{i}=1$, so $\lambda_{i}=1$ for $\bar{\chi}_{i} \in Y$. Then $r_{i}=1$ as required.

Theorem 3.2. Let $G$ be a finitely generated group such that

$$
\Sigma^{1}(G)^{c}=\left\{\left[\chi_{1}\right], \ldots,\left[\chi_{m}\right]\right\}
$$

is finite, nonempty, and contains only discrete points. Let $N=\bigcap_{i} \operatorname{Ker}\left(\chi_{i}\right)$, and let $V=\operatorname{Hom}_{\mathbb{Z}}(G / N, \mathbb{R})$. Suppose that the image of $\left\{\chi_{1}, \ldots, \chi_{m}\right\}$ in $V$ is a basis of $V$ as an $\mathbb{R}$-vector space. Then $R(\varphi)$ is infinite for every $\varphi \in \operatorname{Aut}(G)$, that is, $G$ has the property $R_{\infty}$.

Proof. By the previous lemma, we can fix representatives $\chi_{i}$ (remember we can multiply any $\chi_{i}$ with a positive real number) so that the map $\theta \in \operatorname{End}_{\mathbb{Z}}(V)$, induced 
by $\varphi$, permutes the elements of $\left\{\bar{\chi}_{1}, \ldots, \bar{\chi}_{m}\right\}$, where $\left\{\bar{\chi}_{1}, \ldots, \bar{\chi}_{m}\right\}$ is the image of $\left\{\chi_{1}, \ldots, \chi_{m}\right\}$ in $V$. Then

$$
\theta\left(\bar{\chi}_{1}+\cdots+\bar{\chi}_{m}\right)=\bar{\chi}_{1}+\cdots+\bar{\chi}_{m} \quad \text { and } \quad \bar{\chi}_{1}+\cdots+\bar{\chi}_{m} \neq 0
$$

in $V$, that is, $\theta$ has eigenvalue 1 . Then $R(\bar{\varphi})$ is infinite by the preliminaries from Section 2c; hence $R(\varphi)$ is infinite.

Theorem 3.3. Let $G$ be a finitely generated group such that

$$
\Sigma^{1}(G)^{c}=\left\{\left[\chi_{1}\right], \ldots,\left[\chi_{m}\right]\right\}
$$

is finite, nonempty, and contains only discrete points. Let $N=\bigcap_{i} \operatorname{Ker}\left(\chi_{i}\right)$ and $V=\operatorname{Hom}_{\mathbb{Z}}(G / N, \mathbb{R})$. Suppose that the image of $\left\{\chi_{1}, \ldots, \chi_{m}\right\}$ in $V$ lies in an open half subspace. Then $R(\varphi)$ is infinite for every $\varphi \in \operatorname{Aut}(G)$, that is, $G$ has the property $R_{\infty}$.

Proof. As in the proof of the previous theorem, we can fix representatives $\chi_{i}$ such that $w=\bar{\chi}_{1}+\cdots+\bar{\chi}_{m}$ is fixed by $\theta$, where $\bar{\chi}_{i}$ is the image of $\chi_{i}$ in $V$. Since $\left\{\bar{\chi}_{1}, \ldots, \bar{\chi}_{m}\right\}$ lies in an open half subspace of $V$, we deduce that $w \neq 0$. We continue as in the proof of the previous theorem.

Corollary 3.4. Let $G$ be a finitely generated group such that

$$
\Sigma^{1}(G)^{c}=\left\{\left[\chi_{1}\right], \ldots,\left[\chi_{m}\right]\right\}
$$

is finite, nonempty, and contains only discrete points. Then there is a subgroup of finite index $H$ of $\operatorname{Aut}(G)$ such that $R(\varphi)$ is infinite for every $\varphi \in H$.

Proof. By Lemma 3.1 the image of $\operatorname{Aut}(G)$ in $\operatorname{End}_{\mathbb{Z}}(V)$ permutes the characters $\bar{\chi}_{1}, \bar{\chi}_{2}, \ldots, \bar{\chi}_{m}$, where the coordinates of $\bar{\chi}_{i}$ are integers with maximal common divisor 1 , and $V=\operatorname{Hom}_{\mathbb{Z}}(G / N, \mathbb{R})$ is defined as before. Let $H$ be the kernel of the action of $\operatorname{Aut}(G)$ on $\operatorname{End}_{\mathbb{Z}}(V)$. Then the elements of $H$ fix every $\bar{\chi}_{i}$; in particular the map induced by $\varphi$ on the maximal torsion-free abelian quotient of $G$ has eigenvalue 1 . We continue as in the proof of the previous theorem.

Proposition 3.5. Let $G$ be a finitely generated nilpotent group, and let $\varphi \in \operatorname{Aut}(G)$ be such that $\varphi(g)$ is conjugate to $g$ for some element $g \in G$ of infinite order. Then $R(\varphi)$ is infinite.

Proof. We induct on the nilpotency length of $G$. The case when $G$ is abelian is obvious and well known.

Suppose that $G$ is nonabelian. Let $Z(G)$ be the center of $G$, so $\bar{G}=G / Z(G)$ is of nilpotency length strictly smaller than the nilpotency length of $G$.

Let $\bar{\varphi}$ be the automorphism of $\bar{G}$ induced by $\varphi$. If there is an element of $\bar{G}$ of infinite order that is sent by $\bar{\varphi}$ to a conjugate, then by induction $R(\bar{\varphi})=\infty$, so $R(\varphi)=\infty$. We can assume that there isn't an element of $\bar{G}$ of infinite order 
that is sent by $\bar{\varphi}$ to a conjugate. The conditions of [Gonçalves and Wong 2003, Lemma 2.1] then hold, and hence it suffices to show that $R\left(\varphi^{\prime}\right)=\infty$ to deduce that $R(\varphi)=\infty$, where $\varphi^{\prime}$ is the restriction of $\varphi$ to $N$.

Let $g \in G$ be an element of infinite order such that $\varphi(g) \in g^{G}$. By the preceding paragraph, the image of $g$ in $\bar{G}$ has finite order. If $g \notin Z(G)$, some nontrivial power of $g$ is in $Z(G)$, so we can assume that $g \in Z(G)$. Then $g^{G}=g$ and $\varphi(g)=g$.

Consider one twisted conjugated class

$$
X_{h}=\left\{a h \varphi^{\prime}(a)^{-1}\right\}_{a \in Z(G)} \text { for some } h \in Z(G) .
$$

Because there is an element of infinite order in $Z(G)$ that is fixed by $\varphi^{\prime}$, we see that $\left\{a \varphi^{\prime}(a)^{-1}\right\}_{a \in Z(G)}$ is a subgroup of infinite index in $Z(G)$; hence $X_{h}$ is a coset class of a subgroup of infinite index in $Z(G)$. In particular this shows that we have an infinite number of twisted conjugacy classes $X_{h}$ when $h \in Z(G)$, that is, $R\left(\varphi^{\prime}\right)=\infty$.

\section{Applications}

4a. On the generalized Thompson's group and some soluble groups. For a group $G$ of piecewise linear transformations of the unit interval, we say that $G$ is irreducible if $G$ does not fix a point of $(0,1)$. There are two distinguished real characters $\rho$ and $\lambda$ of $G$ defined by $\lambda(f)=\log _{2} f^{\prime}(0)$ and $\rho(f)=\log _{2} f^{\prime}(1)$.

Theorem 4.1. Let $G$ be a finitely generated, irreducible group of piecewise linear transformations of the unit interval; let $\rho$ and $\lambda$ defined above be nonzero rational nonantipodal characters such that $\lambda(\operatorname{Ker}(\rho))=\operatorname{Im}(\lambda)$ and $\rho(\operatorname{Ker}(\lambda))=\operatorname{Im}(\rho)$. Then $R(\varphi)$ is infinite for every $\varphi \in \operatorname{Aut}(G)$, that is, $G$ has the property $R_{\infty}$.

Proof. By [Bieri et al. 1987, Theorem 8.1], the invariant $\Sigma^{1}(G)^{c}$ is equal to $\{[\rho],[\lambda]\}$, and by hypothesis the conditions of Theorem 3.2 are satisfied.

The generalized Thompson's group $F_{n, 0}$, where $n \geq 2$ (we use the notation of Brin and Guzmán [1998], which differs from the notation in [Brown 1987a]), is defined with the infinite presentation

$$
\left.\left\langle x_{0}, x_{1}, x_{2}, \ldots\right| x_{j}^{-1} x_{i} x_{j}=x_{j+n-1} \text { for } 0 \leq j<i\right\rangle ;
$$

the case $n=2$ is the classical Thompson's group $F$. The groups above were first defined and shown to be of type $F P_{\infty}$ in [Brown 1987a]. Some other groups of PL functions on the interval are known to be of type $F P_{\infty}$ [Stein 1992]. The automorphism group of $F_{n, 0}$ is understood completely only for $n=2$ [Brin 1996], and only some partial results are known for general $n>2$ [Brin and Guzmán 1998]. Although we do not know much about the full automorphism group of $F_{n, 0}$, we can still deduce the following result from Theorem 4.1. This answers a question 
asked in [Bleak et al. 2008], where it was shown that the classical Thompson's group $F$ has the property $R_{\infty}$.

Corollary 4.2. The generalized Thompson's group $F_{n, 0}$ has the property $R_{\infty}$.

Proof. The generalized Thompson's group $F_{n, 0}$ has several presentations as a group of PL functions of different intervals. In [Brin and Guzmán 1998, Lemma 2.3.1], a description of $F_{n, 0}$ is given as the group of PL functions $f$ on the interval $[0, n-1]$, where the set $Y_{f}$ of break points is finite, the image of $Y_{f}$ under $f$ is inside $\mathbb{Z}[1 / n]$, the slopes of all linear functions are integral powers of $n$, and the slopes of $f$ at 0 and $n-1$ can be arbitrary integral powers of $n$. Furthermore $F_{n, 0}$ does not fix a point on the open interval $(0, n-1)$. In that paper, the elements of $F_{n, 0}$ act on the right of the interval, but by setting $f(x)=(x) f^{-1}$, we get an action on the left. By obvious rescaling (that is, a linear map that gives a bijection between the interval $[0, n-1]$ and $[0,1])$, we get a description of $F_{n, 0}$ as a subgroup of PL functions acting on the left of the interval $[0,1]$, and then we can apply Theorem 4.1.

All $\Sigma$-invariants are calculated [Bieri et al. 2010] for the original Thompson's group $F$, but for the generalized ones these invariants are not known except in dimension 1 .

Theorem 4.3. Let $G$ be a nonpolycyclic nilpotent-by-abelian group of type $F P_{\infty}$. Then $R(\varphi)$ is infinite for every $\varphi \in \operatorname{Aut}(G)$, that is, $G$ has the property $R_{\infty}$.

Proof. Soluble groups of type $F P_{\infty}$ are nilpotent-by-abelian-by-finite, so assuming that $G$ is nilpotent-by-abelian is not a very strong restriction.

By the classification of soluble groups of type $F P_{\infty}, G$ is constructible, that is, built from the trivial group by finite and HNN extensions [Kropholler 1993].

Suppose that the commutator $G^{\prime}$ is not finitely generated; hence $\Sigma^{1}(G)^{c}$ is not empty by Theorem 2.1. By [Bieri and Strebel 1982], $\Sigma^{1}(G)^{c}$ is a finite set of discrete points $\left\{\left[\chi_{1}\right], \ldots,\left[\chi_{m}\right]\right\}$ that lies in an open hemisphere of $S(G)$. Then we can apply Theorem 3.3.

Finally if the commutator $G^{\prime}$ is finitely generated, $G$ is polycyclic since it is nilpotent, a contradiction.

A group $G$ is said to be of finite Prüfer rank if there is a number $d$ such that every finitely generated subgroup is generated by at most $d$ elements. In particular if $1 \rightarrow A \rightarrow G \rightarrow Q \rightarrow 1$ is a short exact sequence of groups with $A$ and $Q$ abelian, and $G$ finitely generated, then $G$ has finite Prüfer rank if and only if the torsion part of $A$ is finite and $A \otimes_{\mathbb{Z}} \mathbb{Q}$ is finite-dimensional as a $\mathbb{Q}$-vector space. By the link between valuation theory and $\Sigma_{A}^{c}(Q)$ [Bieri and Groves 1984, Theorem 8.1], by the existing bijection between $\Sigma_{A}^{c}(Q)$ and $\Sigma^{1}(G)^{c}$ explained in the penultimate paragraph of Section $2 \mathrm{~b}$, and because $G$ has finite Prüfer rank, $\Sigma^{1}(G)^{c}$ is a finite set $\left\{\left[\chi_{1}\right], \ldots,\left[\chi_{m}\right]\right\}$, where $\chi_{1}, \ldots, \chi_{m}$ are discrete characters. 
Lemma 4.4. Let $G$ be a finitely generated metabelian group of finite Prüfer rank that is not polycyclic. Then there is a subgroup of finite index $H$ of $\operatorname{Aut}(G)$ such that $R(\varphi)$ is infinite for every $\varphi \in H$.

Proof. Since $G$ is not polycyclic, $G^{\prime}$ is not finitely generated, and so $\Sigma^{1}(G)^{c}$ is not empty by Theorem 2.1. On the other hand $\Sigma^{1}(G)^{c}$ is a finite set of discrete points. Then we can apply Corollary 3.4.

Corollary 4.5. Let $G$ be a finitely generated group such that $G / G^{\prime \prime}$ is of finite Prüfer rank and $G / G^{\prime \prime}$ is not polycyclic. Then there is a subgroup of finite index $H$ of $\operatorname{Aut}(G)$ such that $R(\varphi)$ is infinite for every $\varphi \in H$.

Proof. By the previous lemma there is a subgroup $H_{0}$ of finite index in $\operatorname{Aut}\left(G / G^{\prime \prime}\right)$ such that for every $\varphi_{0} \in H_{0}$ the number $R\left(\varphi_{0}\right)$ is infinite. Then we can define $H$ as the full preimage of $H_{0}$ in $\operatorname{Aut}(G)$.

\section{4b. More on groups with metabelian quotients of type $\mathrm{FP}_{2}$.}

Theorem 4.6. Let $G$ be a finitely generated metabelian group such that $G$ is not polycyclic and $\Sigma^{1}(G)^{c}$ is not a finite union of subspheres. Then there is a subgroup of finite index $H$ of $\operatorname{Aut}(G)$ such that $R(\varphi)$ is infinite for every $\varphi \in H$.

Proof. As before since $G$ is not polycyclic (in this case, this is equivalent to $G^{\prime}$ not being finitely generated) $\Sigma^{1}(G)^{c}$ is not empty by Theorem 2.1 .

We view $A=G^{\prime}$ as a left $\mathbb{Z} Q$-module via conjugation, where $Q=G / G^{\prime}$; we denote this $\mathbb{Z} Q$-action on $A$ by $\circ$, that is, if $q=g A$ and $a \in A$, then $q \circ a=g a g^{-1}$.

Let $P_{1}, \ldots, P_{s}$ be all minimal associated primes of the $\mathbb{Z} Q$-module $A$; see [Bourbaki 1989]. Let $I$ be the annihilator of $\mathbb{Z} Q$ in $A$, that is,

$$
I=\{\lambda \in \mathbb{Z} Q \mid \lambda \circ A=0\} .
$$

Then $P_{1}, \ldots, P_{s}$ are the minimal prime ideals above $I$. Let $\bar{\varphi}$ be the automorphism of $Q=G / G^{\prime}$ induced by $\varphi$. We extend $\bar{\varphi}$ by linearity to a ring endomorphism $\hat{\varphi}$ of $\mathbb{Z} Q$ so that the restriction on $\mathbb{Z}$ is identity, and note that for $\lambda \in I$ we have $0_{A}=\varphi\left(0_{A}\right)=\varphi(\lambda \circ A)=\hat{\varphi}(\lambda) \circ \varphi(A)=\hat{\varphi}(\lambda) \circ A$, so $\hat{\varphi}(I)=I$. Then $\hat{\varphi}$ permutes the finite set of prime ideals $P_{1}, \ldots, P_{s}$. Thus by going down to a subgroup of finite index in $\operatorname{Aut}(G)$, we can assume that $\hat{\varphi}\left(P_{i}\right)=P_{i}$ for every $i$, and so $\hat{\varphi}$ induces an automorphism $\varphi_{i}$ of $R_{i}=\mathbb{Z} Q / P_{i}$. By construction, $\varphi_{i}$ is the identity on the image of $\mathbb{Z}$ in $R_{i}$.

Let $\tilde{\varphi}_{i}$ be the extension of $\varphi_{i}$ to the field of fractions $K_{i}$ of $R_{i}$. Let

$$
Q_{i}=Q / T_{i}, \quad \text { where } T_{i}=\left\{q \in Q \mid q-1 \in P_{i}\right\} .
$$

By [Roseblade 1978, Theorem D] or [Bieri and Groves 1986, corollary on p. 426], the group of automorphisms of $K_{i}$ that fixes the prime subfield $k_{i}$ and permutes the elements of $Q_{i}$ induces a finite group on $B_{i} / A_{i}$, where $B_{i}$ is a minimal subgroup of 
$Q_{i}$ such that $k_{i}\left(B_{i}\right) \subseteq K_{i}$ is a purely transcendental extension with rank that equals the rank of the torsion-free group $Q_{i} / B_{i}$, and $A_{i}$ is the subgroup of elements of $Q_{i}$ that are algebraic over $k_{i}$. The group of automorphisms of $K_{i}$ induces a finite group on $A_{i}$ since the Galois group of a finite field extension is finite. Thus the group of automorphisms of $K_{i}$ that fixes the prime subfield $k_{i}$ and permutes the elements of $Q_{i}$ induces a finite group on $B_{i}$.

Then by going down to a subgroup of finite index $H$ in $\operatorname{Aut}(G)$, that is, $\varphi \in H$, we can assume that $\varphi_{i}$ is trivial on $B_{i}$, that is, for $B_{i}=S_{i} / T_{i}$ with $S_{i} \leq Q$ we have $\varphi(s) \in s T_{i}$ for every $s \in S_{i}$. Then if $B_{i}$ is infinite, the map induced by $\varphi$ on $G / G^{\prime} \otimes_{\mathbb{Z}} \mathbb{Q}$ has eigenvalue 1 , and we can apply the observations from Section 2c. Thus we can assume henceforth that the $B_{i}$ are all finite.

We claim that if $B_{i}$ is finite, then

$$
\Sigma_{\mathbb{Z} Q / P_{i}}^{c}(Q)=\left\{[\chi] \in S(Q) \mid \chi\left(S_{i}\right)=0\right\}=: S\left(Q, S_{i}\right),
$$

where $S_{i}$ is defined as above, that is, $S_{i}$ is the subgroup of $Q$ such that $S_{i} / T_{i}=B_{i}$. Indeed by [Bieri and Groves 1984, Theorem 8.1], the class $[\chi]$ is in $\Sigma_{\mathbb{Z} Q / P_{i}}^{c}(Q)$ if and only if there is a real valuation of $\mathbb{Z} Q / P_{i}$ whose restriction on $Q_{i}=Q / T_{i}$ is induced by $\chi$. Since $K_{i}$ is a purely transcendental extension of $k_{i}\left(B_{i}\right)$ with degree exactly the rank of the torsion-free abelian group $Q_{i} / B_{i}$ for every real valuation $w$ of $k_{i}\left(B_{i}\right)$ and any character $\chi: Q_{i} \rightarrow \mathbb{R}$ such that $\chi$ and $w$ coincide on $B_{i}$, there is a valuation of $K_{i}$ that extends both $w$ and $\chi$. Furthermore any real valuation of $k\left(B_{i}\right)$ sends the finite group $B_{i}$ to zero. Thus any real character of $Q_{i}$ extends to a real valuation of $K_{i}$ and so by restriction to a real valuation of $\mathbb{Z} Q / P_{i}$. Since $T_{i}-1$ maps to 0 in $\mathbb{Z} Q / P_{i}$ and any real valuation of $\mathbb{Z} Q / P_{i}$ sends 0 to infinity for $[\chi] \in \Sigma_{\mathbb{Z} Q / P_{i}}^{c}(Q)$, we always have $\chi\left(T_{i}\right)=\chi(1)=0$. Since $B_{i}$ is finite for every real character $\chi$ such that $\chi\left(T_{i}\right)=0$, we have $\chi\left(S_{i}\right)=0$.

Then as mentioned in Section $2 b$,

$$
\Sigma^{1}(G)^{c}=\Sigma_{A}^{c}(Q)=\Sigma_{\mathbb{Z} Q / I}^{c}(Q)=\bigcup_{i} \Sigma_{\mathbb{Z} Q / P_{i}}^{c}(Q) .
$$

That is, $\Sigma^{1}(G)^{c}$ is a finite union of subspheres $S\left(Q, S_{i}\right)$, a contradiction.

Corollary 4.7. Let $G$ be a group of type $\mathrm{FP}_{2}$. Suppose it does not contain a free subgroup of rank 2 , and $G / G^{\prime \prime}$ is not polycyclic. Then there is a subgroup of finite index $H$ of $\operatorname{Aut}(G)$ such that $R(\varphi)$ is infinite for every $\varphi \in H$.

Proof. By [Bieri and Strebel 1980, Theorem 5.5], the maximal metabelian quotient $G / G^{\prime \prime}$ of $G$ has type $F P_{2}$, and $\Sigma^{1}\left(G / G^{\prime \prime}\right)^{c}$ does not have antipodal points. By Theorem 2.1, since $G / G^{\prime \prime}$ is not polycyclic, $\Sigma^{1}\left(G / G^{\prime \prime}\right)^{c}$ is not empty. Since any subsphere of $S(G)$ has antipodal points, $\Sigma^{1}\left(G / G^{\prime \prime}\right)^{c}$ does not contain a subsphere. Then by Theorem 4.6 there is a subgroup $H_{0}$ of finite index in $\operatorname{Aut}\left(G / G^{\prime \prime}\right)$ such 
that $R\left(\varphi_{0}\right)$ is infinite for every $\varphi_{0} \in H_{0}$. Finally define $H$ to be the full preimage of $H_{0}$ in $\operatorname{Aut}(G)$.

4c. Direct products of groups. The direct product of groups is a particular case of a more general construction: A graph product $G$ of groups is constructed from a graph $\Delta$ and vertex groups $G_{v}$ associated with every vertex $v$ in the set $V$ of vertices of $\Delta$. Then $G$ is the free product of all $G_{v}$ factored by the normal subgroup generated by $\left[G_{v}, G_{w}\right]$, where $v$ and $w$ are any adjacent vertices in $\Delta$.

Theorem 4.8 [Meinert 1995, Theorem A]. Let $G$ be a graph product with a finite underlying graph $\Delta$ and finitely generated vertex groups $\left\{G_{v}\right\}_{v \in V}$. Let $\chi: G \rightarrow \mathbb{R}$ be a nonzero homomorphism. Let $\Delta^{\prime}$ be the full subgraph of $\Delta$ spanned by all vertices $V^{\prime}=\left\{v \in V \mid \chi\left(G_{v}\right) \neq 0\right\}$. Then $[\chi] \in \Sigma^{1}(G)$ if and only if one of the following conditions holds:

(1) If $\Delta^{\prime}=\left\{v_{0}\right\}$, then $\left[\left.\chi\right|_{G_{v_{0}}}\right] \in \Sigma^{1}\left(G_{v_{0}}\right)$, and each vertex of $V \backslash\left\{v_{0}\right\}$ is adjacent to $v_{0}$.

(2) If $\Delta^{\prime}$ has at least 2 vertices, then it is connected, and each vertex of $V \backslash V^{\prime}$ is adjacent to some vertex of $\Delta^{\prime}$.

If applied to a finite graph $\Delta$ in which every pair of vertices is linked by an edge, then this theorem gives a simple formula for the $\Sigma^{1}$ invariant of a direct product of finitely many groups. There were some attempts to prove analogues for higher-dimensional invariants $\Sigma^{m}(G, \mathbb{Z})$ and $\Sigma^{m}(G)$ for $G$ a direct product of groups (such analogues are called direct product conjectures). These conjectures turn out to be false in both homological and homotopical versions [Meier et al. 1998; Schütz 2008], but hold if we consider invariants with coefficients in a field [Bieri and Geoghegan 2010].

Corollary 4.9. Let $H=G_{1} \times \cdots \times G_{m}$, and let $\Sigma^{1}\left(G_{i}\right)^{c}$ be finite (possibly empty) and contain only rational points (if any) for all $1 \leq i \leq n$. Suppose further that $\Sigma^{1}\left(G_{i}\right)^{c}$ is not empty for at least one $i$. Then there is a subgroup of finite index $H_{0}$ of $\operatorname{Aut}(H)$ such that $R(\varphi)$ is infinite for every $\varphi \in H_{0}$.

Proof. By Theorem 4.8, $\Sigma^{1}(G)^{c}$ is finite, nonempty, and contains only discrete points. Thus we can apply Corollary 3.4.

Corollary 4.10. Let $H=G_{1} \times \cdots \times G_{m}$, where $G_{i}$ are generalized Thompson's groups (not necessarily isomorphic). Then $H$ has the property $R_{\infty}$.

Proof. Let $\rho_{i}$ and $\lambda_{i}$ be the characters of $H$ that are zero when restricted to $G_{j}$ for $j \neq i$, and are the classical $\rho$ and $\lambda$ from Section 4 when restricted to $G_{i}$. By Theorem 4.8, $\Sigma^{1}(H)^{c}=\left\{\left[\lambda_{i}\right],\left[\rho_{i}\right]\right\}_{1 \leq i \leq m}$. Then we can apply Theorem 3.2. 
Examples. Here we exhibit groups $G$ that have abelianization $\mathbb{Z}$ and nonempty $\Sigma^{1}(G)^{c}$. Let $G$ be a knot group with commutator $G^{\prime}$ infinitely generated. For every knot group, we have $G / G^{\prime} \simeq \mathbb{Z}$. By Theorem 2.1, since $G^{\prime}$ is infinitely generated, $\Sigma^{1}(G) \neq S(G)$.

Let $D$ be the double of $G$, that is, we make the free amalgamated product $G *_{\mathbb{Z}^{2}} G$, where $\mathbb{Z}^{2}$ is the torus subgroup of $G$. Thus $D$ is a 3-manifold group and $D / D^{\prime} \simeq \mathbb{Z}$. By the results of Thurston [1986] for 3-manifold groups and their translation into algebraic language [Bieri et al. 1987, Corollary F], we have $\Sigma^{1}(D)=-\Sigma^{1}(D)$, and $\Sigma^{1}(D)$ is nonempty if and only if the 3-manifold fibers over $S^{1}$; in our case this means that $D^{\prime}$ is finitely generated. This obviously does not hold as $G$ is a quotient of $D$, and $G^{\prime}$ is not finitely generated. Then $\Sigma^{1}(D)$ is empty. Since $D / D^{\prime} \simeq \mathbb{Z}$, we see that $\Sigma^{1}(D)^{c}=S(D)$ has 2 elements.

4d. The pure symmetric automorphism group. In this section, $G$ is the pure symmetric automorphism group of the free group $F$ with basis $\left\{x_{1}, \ldots, x_{n}\right\}$. It is generated by $\left\{\alpha_{i, j}\right\}_{1 \leq i \neq j \leq n}$, where

$$
\alpha_{i, j}\left(x_{i}\right)=x_{j}^{-1} x_{i} x_{j} \quad \text { and } \quad \alpha_{i, j}\left(x_{k}\right)=x_{k} \quad \text { for } k \neq i .
$$

By [McCool 1986], $G$ is finitely presented with relations

$$
\begin{aligned}
{\left[\alpha_{i, j}, \alpha_{k, l}\right] } & =1 & & \text { if } i, j, k, l \text { are different }, \\
{\left[\alpha_{i, j}, \alpha_{k, j}\right] } & =1 \quad \text { and }\left[\alpha_{i, j} \alpha_{k, j}, \alpha_{i, k}\right]=1 & & \text { if } i, j, k \text { are different. }
\end{aligned}
$$

By [Brady et al. 2001], $G$ is a duality group of dimension $n-1$. By the main result of [Orlandi-Korner 2000], the class $[\chi]$ is in $\Sigma^{1}(G)^{c}$ if and only if exactly one of the following holds:

(1) $\chi\left(\alpha_{i, j}\right), \chi\left(\alpha_{j, i}\right) \in \mathbb{R}$ for some $1 \leq i<j \leq n$ and $\chi\left(\alpha_{r, s}\right)=0$ for the remaining indices;

(2) $\chi\left(\alpha_{j, i}\right)=-\chi\left(\alpha_{k, i}\right), \chi\left(\alpha_{i, k}\right)=-\chi\left(\alpha_{j, k}\right)$, and $\chi\left(\alpha_{k, j}\right)=-\chi\left(\alpha_{i, j}\right)$ for some $1 \leq i<j<k \leq n$ and $\chi\left(\alpha_{r, s}\right)=0$ for the remaining indices.

We write $A_{i, j}$ for the set of characters of first type and $B_{i, j, k}$ for the set of second type (in both cases including the trivial character). Note that $A_{i, j}$ and $B_{i, j, k}$ are $\mathbb{R}$-subspaces of $\operatorname{Hom}_{\mathbb{Z}}(G, \mathbb{R})$ of dimensions 2 and 3, respectively.

Theorem 4.11. Let $G$ be the pure symmetric automorphism group of a free group of rank $n$. Then there is a subgroup $H$ of finite index in $\operatorname{Aut}(G)$ such that $R(\varphi)$ is infinite for every $\varphi \in H$.

Proof. Since $\operatorname{Aut}(G)$ permutes

$$
W=\left(\bigcup_{1 \leq i<j \leq n} A_{i, j}\right) \cup\left(\bigcup_{1 \leq i<j<k \leq n} B_{i, j, k}\right) \subset \operatorname{Hom}_{\mathbb{Z}}(G, \mathbb{R}),
$$


and the only 3-dimensional spaces inside $W$ are the $B_{i, j, k}$, we see that $\operatorname{Aut}(G)$ permutes the spaces $B_{i, j, k}$ and thus also the elements of $M=\bigcup_{1 \leq i<j \leq n} A_{i, j}$. Also, the only 2-dimensional spaces inside $M$ are the $A_{i, j}$, so $\operatorname{Aut}(G)$ permutes the spaces $A_{i, j}$. By going down to a subgroup of finite index in $\operatorname{Aut}(G)$, we can consider only automorphisms $\varphi$ of $G$ such that $\varphi$ sends every $A_{i, j}$ to $A_{i, j}$ and every $B_{i, j, k}$ to $B_{i, j, k}$.

Let $\left\{\alpha_{i, j}^{*}\right\}_{i, j}$ be the basis of $\operatorname{Hom}(G, \mathbb{R})$ dual to $\left\{\alpha_{i, j}\right\}_{i, j}$ and $\varphi^{*}$ be the automorphism of $\operatorname{Hom}(G, \mathbb{R})$ induced by $\varphi$. Note that

$$
B_{i, j, k}=\mathbb{R}\left(\alpha_{i, j}^{*}-\alpha_{k, j}^{*}\right)+\mathbb{R}\left(\alpha_{i, k}^{*}-\alpha_{j, k}^{*}\right)+\mathbb{R}\left(\alpha_{k, i}^{*}-\alpha_{j, i}^{*}\right)
$$

and $B_{i, j, k}$ is invariant under $\varphi^{*}$. Then

$$
\varphi^{*}\left(\alpha_{i, j}^{*}-\alpha_{k, j}^{*}\right) \in B_{i, j, k} .
$$

On the other hand, since $A_{i, j}$ and $A_{k, j}$ are invariant under $\varphi^{*}$, we have

$$
\varphi^{*}\left(\alpha_{i, j}^{*}\right) \in \mathbb{R} \alpha_{i, j}^{*}+\mathbb{R} \alpha_{j, i}^{*} \quad \text { and } \quad \varphi^{*}\left(\alpha_{k, j}^{*}\right) \in \mathbb{R} \alpha_{k, j}^{*}+\mathbb{R} \alpha_{j, k}^{*} ;
$$

hence

$$
\varphi^{*}\left(\alpha_{i, j}^{*}-\alpha_{k, j}^{*}\right) \in \mathbb{R} \alpha_{i, j}^{*}+\mathbb{R} \alpha_{j, i}^{*}+\mathbb{R} \alpha_{k, j}^{*}+\mathbb{R} \alpha_{j, k}^{*} .
$$

Combining (4-1) and (4-3), we get

$$
\varphi^{*}\left(\alpha_{i, j}^{*}-\alpha_{k, j}^{*}\right)=a \alpha_{i, j}^{*}-a \alpha_{k, j}^{*} \quad \text { for some } a \in \mathbb{R} .
$$

Then by (4-2),

$$
\varphi^{*}\left(\alpha_{i, j}^{*}\right)=a \alpha_{i, j}^{*} \quad \text { and } \quad \varphi^{*}\left(\alpha_{k, j}^{*}\right)=a \alpha_{k, j}^{*} .
$$

In particular, $\varphi$ induces a diagonal linear map $\bar{\varphi}$ on the abelianization $G / G^{\prime}$, and since $\varphi$ is invertible, the product of eigenvalues is either 1 or -1 , and all $a$ are integers. Then $a$ is either 1 or -1 , and either $\bar{\varphi}$ has eigenvalue 1 and we are done by the remarks from Section $2 \mathrm{c}$, or $\bar{\varphi}$ is $-\mathrm{id}_{G / G^{\prime}}$. By going to a subgroup of index 2, we can avoid the last case.

4e. Houghton groups. Let $\mathbb{N}=\{1,2, \ldots\}$ be the set of the natural numbers, and let $M=\{1,2, \ldots, n\} \times \mathbb{N}$ for $n \in \mathbb{N}$. The Houghton group $H_{n}$ consists of all permutations $g$ of $M$ such that there is an $n$-tuple $\left(m_{1}, \ldots, m_{n}\right) \in \mathbb{Z}^{n}$ such that $g(i, r)=\left(i, r+m_{i}\right)$ for all $i \in\{1, \ldots, n\}$ and all but finitely many $(i, r) \in M$. These groups were first introduced in [Houghton 1978]. There is an important homomorphism $f: H_{n} \rightarrow \mathbb{Z}^{n}$ sending each permutation $g$ to the corresponding $n$-tuple $\left(m_{1}, \ldots, m_{n}\right)$. We always have $\sum_{1 \leq i \leq n} m_{i}=0$.

For $n \geq 2$, the group $H_{n}$ is finitely generated. More generally K. Brown [1987a] has shown that $H_{n}$ is of type $F P_{n-1}$ but not of type $F P_{n}$. For $n \geq 2$, the group $H_{n}$ 
is generated by $\left\{t_{2}, \ldots, t_{n}\right\}$, where

$$
\begin{array}{lll}
t_{i}(j, r)=(j, r) & \text { for } i \neq j, j \neq 1 & t_{i}(i, 1)=(1,1), \\
t_{i}(i, r)=(i, r-1) & \text { for } r>1, & t_{i}(1, r)=(1, r+1) .
\end{array}
$$

Also $H_{n} /\left[H_{n}, H_{n}\right] \simeq \mathbb{Z}^{n-1}$; see [Gehrke 1998]. The invariant $\Sigma^{1}\left(H_{n}\right)^{c}$ for $n \geq 2$ was calculated in [Brown 1987b] as

$$
\Sigma^{1}\left(H_{n}\right)^{c}=\left\{\left[\alpha_{1}\right], \ldots,\left[\alpha_{n}\right]\right\},
$$

where $\alpha_{i}=-\pi_{i} f$ and $\pi_{i}: \mathbb{Z}^{n} \rightarrow \mathbb{Z}$ is the projection to the $i$-th coordinate. Thus $\Sigma^{1}\left(H_{n}\right)^{c}$ is a finite set of rational points of $S\left(H_{n}\right)$, so we can apply Corollary 3.4 and get the following result.

Corollary 4.12. Let $n \geq 2$ be a natural number, and let $G=H_{n}$. Then there is a subgroup $H$ of finite index in $\operatorname{Aut}(G)$ such that $R(\varphi)$ is infinite for every $\varphi \in H$.

\section{Limit groups}

In the previous section we saw that sometimes to understand the structure of $\Sigma^{1}(G)$, it suffices to study the twisted conjugacy classes, although we might not have a complete classification of the whole automorphism group. In this section we study twisted conjugacy classes for 1-ended limit groups $G$. Kochloukova [2010] proved that $\Sigma^{1}(G)$ is empty for any limit group $G$, so we have to find a different approach to study the Reidemeister number $R(\varphi)$. As noted in the introduction, the class of limit groups coincides with the class of finitely generated fully residually free groups.

There are results about the automorphism group of 1-ended limit groups that give a description of a subgroup of finite index of $\operatorname{Aut}(G)$ [Bumagin et al. 2007]. In the general case (when the limit group $G$ can be decomposed as a free product), it is known that $\operatorname{Out}(G)$ has a subgroup of finite index that has a finite classifying space [Guirardel and Levitt 2007, Theorem 6.5]. Here we do not need the full strength of these results, but use the existence of a canonical abelian JSJ decomposition for a 1-ended limit group $G$ that is neither a surface group nor free abelian of finite rank [Bumagin et al. 2007, Theorem 3.13]. This means that $G$ is the fundamental group of a finite graph of groups with finitely generated abelian edge stabilizers, that is, the group associated to each edge is abelian and there is a canonical graph of groups with these properties. There are limited possibilities for the vertex groups: abelian groups of finite rank, HQ-groups (that is, surface groups), and rigid groups [Bumagin et al. 2007].

The uniqueness of the canonical abelian JSJ decomposition shows that there is a subgroup $H_{0}$ of finite index in $\operatorname{Aut}(G)$ such that under an automorphism $\varphi \in H_{0}$ a vertex group goes to a conjugate of a vertex group. Also, up to conjugation and 
generalized Dehn twists along edges (these twists are defined in [Bumagin et al. 2007, Definition 2.4] and have the property that on a fixed vertex group they act by conjugation with an element of $G$ ), an automorphism $\varphi \in H_{0}$ induces isomorphisms on the vertex groups that permute the adjacent edge groups up to conjugation.

The proof of the following result requires the existence of a canonical JSJ decomposition of $G$ that has bipartite structure as defined in [Bumagin et al. 2007, Theorem 3.13]. This requires that $G$ is a 1-ended limit group that is neither a free abelian nor a surface group.

Theorem 5.1. Let $G$ be a 1-ended limit group that is neither a free abelian nor a surface group. Then there is a subgroup of finite index $H$ of $\operatorname{Aut}(G)$ such that $R(\varphi)$ is infinite for every $\varphi \in H$.

Proof. Consider the canonical abelian JSJ decomposition of $G$. Then there is a subgroup of finite index $H_{0}$ of $\operatorname{Aut}(G)$ such that for every $\varphi \in H_{0}$ and every $t \in V(\Gamma) \cup E(\Gamma)$, we have $\varphi\left(G_{t}\right)=G_{t}^{g_{t}}$ for some $g_{t} \in G$. Note that $H_{0}=\operatorname{Aut}(G)$ if the underlying graph of groups $\Gamma$ of the JSJ decomposition of $G$ does not have nontrivial symmetries that permute vertices and edges with isomorphic vertex and edge groups, respectively.

We are particularly interested in edge groups. Since the underlying graph is bipartite, every nonabelian group is linked with an abelian one. Two nonabelian groups or two abelian groups are not directly linked via an edge in the canonical JSJ decomposition. Thus if the connected graph $\Gamma$ has more than one vertex, there is a nonabelian vertex group, and if $\Gamma$ has just one vertex, the unique vertex group cannot be abelian as $G$ is nonabelian. The nonabelian groups are divided into two classes: rigid and flexible.

By going down to a subgroup of finite index $H_{1}$ in $H_{0}$, we can assume that, up to conjugation and generalized Dehn twists along edges, the elements of $H_{1}$ that fix any rigid vertex group (not pointwise but as a group) are the identity on this rigid group [Kharlampovich and Myasnikov 2005, Theorem 15.1; Bumagin et al. 2007, Corollary 4.10]. Furthermore the edge groups of flexible vertices (that is, $H Q$ vertices) are infinite cyclic [Bumagin et al. 2007, Definition 3.9], and $\operatorname{Aut}(\mathbb{Z})=\mathbb{Z}_{2}$ is finite. Then by further going down to a subgroup $H$ of finite index in $H_{1}$, we can consider only automorphisms of $G$ that up to conjugation and generalized Dehn twists along edges send an edge group of a flexible vertex group identically to itself. Thus

$$
\varphi(g) \in g^{G} \text { for every } \varphi \in H \text { and every } g \neq 1 \text { of an edge group. }
$$

Let $\gamma_{i}(G)$ be the $i$-th term of the lower central series of $G$, and let $t_{i}(G)$ be the normal subgroup of $G$ containing $\gamma_{i}(G)$ such that $G / t_{i}(G)$ is the maximal torsionfree quotient of $G / \gamma_{i}(G)$. Kochloukova [2010] showed that $\bigcap_{i \geq 1} t_{i}(G)=1$, an 
easy corollary of the fact that $G$ is fully residually free. In other words, for every finite subset $T$ of $G$ there is a homomorphism from $G$ to a free group that is injective on $T$. Then for the nontrivial element $g$ from (5-1) we can find some $i$ such that $g \notin t_{i}(G)$. Thus $\varphi$ induces an automorphism $\bar{\varphi}$ on $Q=G / t_{i}(G)$ that sends the image $\bar{g}$ of $g$ in $Q$ to some conjugate of $\bar{g}$ in $Q$. Then $R(\bar{\varphi})$ is infinite by Proposition 3.5; hence $R(\varphi)$ is infinite.

\section{Acknowledgments}

The second author thanks Olga Kharlampovich for conversations during the school of algebra in Rio de Janeiro in 2008 about her results on the automorphism group of 1-ended limit groups [Bumagin et al. 2007].

\section{References}

[Alibegović and Bestvina 2006] E. Alibegović and M. Bestvina, "Limit groups are CAT(0)", J. London Math. Soc. (2) 74:1 (2006), 259-272. MR 2007m:57004

[Bestvina and Brady 1997] M. Bestvina and N. Brady, "Morse theory and finiteness properties of groups", Invent. Math. 129:3 (1997), 445-470. MR 98i:20039 Zbl 0888.20021

[Bieri and Geoghegan 2010] R. Bieri and R. Geoghegan, "Sigma invariants of direct products of groups", Groups Geom. Dyn. 4:2 (2010), 251-261. MR 2595091 Zbl 05696864

[Bieri and Groves 1984] R. Bieri and J. R. J. Groves, "The geometry of the set of characters induced by valuations", J. Reine Angew. Math. 347 (1984), 168-195. MR 86c:14001 Zbl 0526.13003

[Bieri and Groves 1986] R. Bieri and J. R. J. Groves, "A rigidity property for the set of all characters induced by valuations", Trans. Amer. Math. Soc. 294:2 (1986), 425-434. MR 87i:16015 Zbl 0598.13001

[Bieri and Renz 1988] R. Bieri and B. Renz, "Valuations on free resolutions and higher geometric invariants of groups", Comment. Math. Helv. 63:1 (1988), 464-497. MR 90a:20106 Zbl 0654.20029

[Bieri and Strebel 1980] R. Bieri and R. Strebel, "Valuations and finitely presented metabelian groups", Proc. London Math. Soc. (3) 41:3 (1980), 439-464. MR 81j:20080 Zbl 0448.20029

[Bieri and Strebel 1981] R. Bieri and R. Strebel, "A geometric invariant for modules over an abelian group”, J. Reine Angew. Math. 322 (1981), 170-189. MR 82f:20017 Zbl 0448.20010

[Bieri and Strebel 1982] R. Bieri and R. Strebel, "A geometric invariant for nilpotent-by-abelian-byfinite groups”, J. Pure Appl. Algebra 25:1 (1982), 1-20. MR 83i:20031 Zbl 0485.20026

[Bieri et al. 1987] R. Bieri, W. D. Neumann, and R. Strebel, "A geometric invariant of discrete groups", Invent. Math. 90:3 (1987), 451-477. MR 89b:20108 Zbl 0642.57002

[Bieri et al. 2010] R. Bieri, R. Geoghegan, and D. H. Kochloukova, "The Sigma invariants of Thompson's group F", Groups Geom. Dyn. 4:2 (2010), 263-273. Zbl 05696865

[Bleak et al. 2008] C. Bleak, A. Fel'shtyn, and D. L. Gonçalves, "Twisted conjugacy classes in R. Thompson's group F", Pacific J. Math. 238:1 (2008), 1-6. MR 2009g:20056

[Bourbaki 1989] N. Bourbaki, Commutative algebra, Elements of Mathematics 2.2, Springer, Berlin, 1989. MR 90a:13001 Zbl 0666.13001

[Brady et al. 2001] N. Brady, J. McCammond, J. Meier, and A. Miller, "The pure symmetric automorphisms of a free group form a duality group", J. Algebra 246 (2001), 881-896. MR 2002i:20036 Zbl 0995.20010 
[Brin 1996] M. G. Brin, "The chameleon groups of Richard J. Thompson: automorphisms and dynamics", Inst. Hautes Études Sci. Publ. Math. 84:1 (1996), 5-33. MR 99e:57003 Zbl 0891.57037

[Brin and Guzmán 1998] M. G. Brin and F. Guzmán, "Automorphisms of generalized Thompson groups", J. Algebra 203:1 (1998), 285-348. MR 99d:20056 Zbl 0930.20039

[Brown 1987a] K. S. Brown, "Finiteness properties of groups", J. Pure Appl. Algebra 44:1-3 (1987), 45-75. MR 88m:20110 Zbl 0613.20033

[Brown 1987b] K. S. Brown, "Trees, valuations, and the Bieri-Neumann-Strebel invariant", Invent. Math. 90:3 (1987), 479-504. MR 89e:20060 Zbl 0663.20033

[Bumagin et al. 2007] I. Bumagin, O. Kharlampovich, and A. Miasnikov, "The isomorphism problem for finitely generated fully residually free groups", J. Pure Appl. Algebra 208:3 (2007), 961977. MR 2007j:20037 Zbl 1121.20026

[Fel'shtyn 2001] A. L. Fel'shtyn, “The Reidemeister number of any automorphism of a Gromov hyperbolic group is infinite", Zap. Nauchn. Sem. S.-Peterburg. Otdel. Mat. Inst. Steklov. (POMI), Geom. i Topol. 279:6 (2001), 229-240, 250. In Russian; translated in J. Math. Sci. 119:1, (2004), 117-123. MR 2002e:20081

[Fel'shtyn et al. 2008a] A. Fel'shtyn, Y. Leonov, and E. Troitsky, "Twisted conjugacy classes in saturated weakly branch groups", Geom. Dedicata 134:1 (2008), 61-73. MR 2009g:20095

[Fel'shtyn et al. 2008b] A. L. Fel'shtyn, D. L. Gonçalves, and P. Wong, “Twisted conjugacy classes for polyfree groups", preprint, 2008. arXiv 0802.2937

[Gehrke 1998] R. Gehrke, "The higher geometric invariants for groups with sufficient commutativity”, Comm. Algebra 26:4 (1998), 1097-1115. MR 99b:20072 Zbl 0905.20024

[Gersten 1999] S. M. Gersten, "Introduction to hyperbolic and automatic groups", pp. 45-70 in Summer school in group theory (Banff, 1996), edited by O. Kharlampovich, CRM Proc. Lecture Notes 17, American Mathematical Society, Providence, RI, 1999. MR 2000a:20093 Zbl 0926.20028

[Gonçalves 1998] D. L. Gonçalves, "Coincidence Reidemeister classes on nilmanifolds and nilpotent fibrations", Topology Appl. 83:3 (1998), 169-186. MR 98m:55003 Zbl 0929.55002

[Gonçalves and Wong 2003] D. Gonçalves and P. Wong, "Twisted conjugacy classes in exponential growth groups”, Bull. London Math. Soc. 35:2 (2003), 261-268. MR 2003j:20054 Zbl 1030.20020

[Guirardel and Levitt 2007] V. Guirardel and G. Levitt, "The outer space of a free product", Proc. Lond. Math. Soc. (3) 94:3 (2007), 695-714. MR 2008c:20047 Zbl 1168.20011

[Houghton 1978] C. H. Houghton, "The first cohomology of a group with permutation module coefficients", Arch. Math. (Basel) 31:1 (1978), 254-258. MR 80c:20073 Zbl 0377.20044

[Jiang 1983] B. J. Jiang, Lectures on Nielsen fixed point theory, Contemporary Mathematics 14, American Mathematical Society, Providence, RI, 1983. MR 84f:55002 Zbl 0512.55003

[Kharlampovich and Myasnikov 2005] O. Kharlampovich and A. G. Myasnikov, "Effective JSJ decompositions", pp. 87-212 in Groups, languages, algorithms, edited by A. V. Borovik, Contemp. Math. 378, American Mathematical Society, 2005. MR 2006m:20045 Zbl 1093.20019

[Kochloukova 2010] D. H. Kochloukova, "On subdirect products of type $\mathrm{FP}_{m}$ of limit groups", $J$. Group Theory 13:1 (2010), 1-19. MR 2604842 Zbl 05673300

[Kropholler 1993] P. H. Kropholler, "On groups of type (FP) $)_{\infty}$, J. Pure Appl. Algebra 90:1 (1993), 55-67. MR 94j:20051b Zbl 0816.20042

[Levitt 2007] G. Levitt, "On the automorphism group of generalized Baumslag-Solitar groups", Geom. Topol. 11 (2007), 473-515. MR 2008h:20061 Zbl 1143.20014

[Levitt and Lustig 2000] G. Levitt and M. Lustig, "Most automorphisms of a hyperbolic group have very simple dynamics", Ann. Sci. École Norm. Sup. (4) 33:4 (2000), 507-517. MR 2002d:20066 Zbl 0997.20043 
[McCool 1986] J. McCool, "On basis-conjugating automorphisms of free groups", Canad. J. Math. 38:6 (1986), 1525-1529. MR 87m:20093 Zbl 0613.20024

[Meier et al. 1998] J. Meier, H. Meinert, and L. VanWyk, "Higher generation subgroup sets and the $\Sigma$-invariants of graph groups", Comment. Math. Helv. 73:1 (1998), 22-44. MR 99f:57002 Zbl 0899.57001

[Meinert 1995] H. Meinert, "The Bieri-Neumann-Strebel invariant for graph products of groups", J. Pure Appl. Algebra 103:2 (1995), 205-210. MR 96i:20047 Zbl 0838.20027

[Orlandi-Korner 2000] L. A. Orlandi-Korner, "The Bieri-Neumann-Strebel invariant for basis-conjugating automorphisms of free groups", Proc. Amer. Math. Soc. 128:5 (2000), 1257-1262. MR 2000k:20046 Zbl 0963.20016

[Roseblade 1978] J. E. Roseblade, "Prime ideals in group rings of polycyclic groups", Proc. London Math. Soc. (3) 36:3 (1978), 385-447. MR 58 \#10996a Zbl 0391.16008

[Schütz 2008] D. Schütz, "On the direct product conjecture for sigma invariants", Bull. Lond. Math. Soc. 40:4 (2008), 675-684. MR 2009j:20075

[Stein 1992] M. Stein, "Groups of piecewise linear homeomorphisms", Trans. Amer. Math. Soc. 332:2 (1992), 477-514. MR 92k:20075 Zbl 0798.20025

[Taback and Wong 2007] J. Taback and P. Wong, "Twisted conjugacy and quasi-isometry invariance for generalized solvable Baumslag-Solitar groups", J. Lond. Math. Soc. (2) 75:3 (2007), 705-717. MR 2008h:20043 Zbl 1128.20030

[Thurston 1986] W. P. Thurston, "A norm for the homology of 3-manifolds", pp. i-vi and 99-130 Mem. Amer. Math. Soc. 339, American Mathematical Society, 1986. MR 88h:57014

Received January 22, 2009. Revised December 13, 2009.

\section{DACIBERG GONÇALVES}

DePARTAMENTO DE MATEMÁticA - IME-USP

UNIVERSIDAdE DE SÃo PAULO

CAIXA POSTAL 66281

AgÊnCIa Cidade de São Paulo

05314-970 SÃo PAULO, SP

BRAZIL

dlgoncal@ime.usp.br

DESSISLAVA Hristova Kochloukova

DEPARTAMENTO DE MATEMÁtica

UNICAMP

13083-970 CAMPINAS, SP

BRAZIL

desi@ime.unicamp.br 


\title{
PACIFIC JOURNAL OF MATHEMATICS
}

\author{
http://www.pjmath.org \\ Founded in 1951 by \\ E. F. Beckenbach (1906-1982) and F. Wolf (1904-1989)
}

\section{EDITORS}

V. S. Varadarajan (Managing Editor)

Department of Mathematics

University of California

Los Angeles, CA 90095-1555

pacific@math.ucla.edu

Vyjayanthi Chari

Department of Mathematics

University of California

Riverside, CA 92521-0135

chari@math.ucr.edu

Robert Finn

Department of Mathematics Stanford University

Stanford, CA 94305-2125

finn@math.stanford.edu

Kefeng Liu

Department of Mathematics

University of California

Los Angeles, CA 90095-1555

liu@math.ucla.edu
Darren Long

Department of Mathematics

University of California

Santa Barbara, CA 93106-3080

long@math.ucsb.edu

Jiang-Hua Lu

Department of Mathematics

The University of Hong Kong

Pokfulam Rd., Hong Kong jhlu@maths.hku.hk

Alexander Merkurjev

Department of Mathematics

University of California

Los Angeles, CA 90095-1555

merkurev@math.ucla.edu
Sorin Popa

Department of Mathematics University of California

Los Angeles, CA 90095-1555 popa@math.ucla.edu

Jie Qing

Department of Mathematics

University of California

Santa Cruz, CA 95064

qing@cats.ucsc.edu

Jonathan Rogawski

Department of Mathematics

University of California

Los Angeles, CA 90095-1555

jonr@math.ucla.edu

\section{PRODUCTION}

pacific@math.berkeley.edu

\begin{abstract}
Silvio Levy, Scientific Editor Matthew Cargo, Senior Production Editor
\end{abstract}
ACADEMIA SINICA, TAIPEI

CALIFORNIA INST. OF TECHNOLOGY

INST. DE MATEMÁTICA PURA E APLICADA

KEIO UNIVERSITY

MATH. SCIENCES RESEARCH INSTITUTE

NEW MEXICO STATE UNIV.

OREGON STATE UNIV.

\section{SUPPORTING INSTITUTIONS}

STANFORD UNIVERSITY
UNIV. OF BRITISH COLUMBIA
UNIV. OF CALIFORNIA, BERKELEY
UNIV. OF CALIFORNIA, DAVIS
UNIV. OF CALIFORNIA, LOS ANGELES
UNIV. OF CALIFORNIA, RIVERSIDE
UNIV. OF CALIFORNIA, SAN DIEGO
UNIV. OF CALIF., SANTA BARBARA

UNIV. OF CALIF., SANTA CRUZ

UNIV. OF MONTANA

UNIV. OF OREGON

UNIV. OF SOUTHERN CALIFORNIA

UNIV. OF UTAH

UNIV. OF WASHINGTON

WASHINGTON STATE UNIVERSITY

These supporting institutions contribute to the cost of publication of this Journal, but they are not owners or publishers and have no responsibility for its contents or policies.

See inside back cover or www.pjmath.org for submission instructions.

The subscription price for 2010 is US \$420/year for the electronic version, and \$485/year for print and electronic.

Subscriptions, requests for back issues from the last three years and changes of subscribers address should be sent to Pacific Journal of Mathematics, P.O. Box 4163, Berkeley, CA 94704-0163, U.S.A. Prior back issues are obtainable from Periodicals Service Company, 11 Main Street, Germantown, NY 12526-5635. The Pacific Journal of Mathematics is indexed by Mathematical Reviews, Zentralblatt MATH, PASCAL CNRS Index, Referativnyi Zhurnal, Current Mathematical Publications and the Science Citation Index.

The Pacific Journal of Mathematics (ISSN 0030-8730) at the University of California, c/o Department of Mathematics, 969 Evans Hall, Berkeley, CA 94720-3840, is published monthly except July and August. Periodical rate postage paid at Berkeley, CA 94704, and additional mailing offices. POSTMASTER: send address changes to Pacific Journal of Mathematics, P.O. Box 4163, Berkeley, CA 94704-0163.

PJM peer review and production are managed by EditFLOW ${ }^{\mathrm{TM}}$ from Mathematical Sciences Publishers.

PUBLISHED BY PACIFIC JOURNAL OF MATHEMATICS

at the University of California, Berkeley 94720-3840

A NON-PROFIT CORPORATION

Typeset in LATEX

Copyright $(\mathrm{C} 2010$ by Pacific Journal of Mathematics 


\section{PACIFIC JOURNAL OF MATHEMATICS}

Volume $247 \quad$ No. $2 \quad$ October 2010

A family of representations of braid groups on surfaces

257

ByUnG HeE AN and Ki HyOUNG Ko

Parametrization of holomorphic Segre-preserving maps

283

R. BLAIR ANGLE

Chern classes on differential $K$-theory

ULRICH BUNKE

Laplacian spectrum for the nilpotent Kac-Moody Lie algebras

DMITRY FUCHS and CONSTANCE WILMARTH

Sigma theory and twisted conjugacy classes

DACIBERG GONÇALVES and DESSISLAVA Hristova Kochloukova

Properties of annular capillary surfaces with equal contact angles

JAMES GORDON and DAVID SIEGEL

Approximating annular capillary surfaces with equal contact angles

JAMES GORDON and DAVID SIEGEL

Harmonic quasiconformal self-mappings and Möbius transformations of the unit ball

David Kalaj and Miodrag S. Mateljević

Klein bottle and toroidal Dehn fillings at distance 5

SANGYOP LEE

Representations of the two-fold central extension of $\mathrm{SL}_{2}\left(\mathbb{Q}_{2}\right)$

HUNG YEAN LOKE and GORDAN SAVIN

Large quantum corrections in mirror symmetry for a 2-dimensional

Lagrangian submanifold with an elliptic umbilic

Giovanni MARELLI

Crossed pointed categories and their equivariantizations

DEEPAK NAIDU

Painlevé analysis of generalized Zakharov equations

Hassan A. ZeDan and Salma M. Al-Tuwairqi 